\title{
Monitorización de niveles plasma de antipsicóticos atípi-
} cos.

Atypical antipsychotics plasma level monitoring.

\author{
Ernesto José Verdura Vizcaíno. Médico Residente a , Daniel Ballesteros Sanz. Médico Residente b, \\ Javier Sanz-Fuentenebro. Médico Adjunto ${ }^{c}$.
}

${ }^{a}$ Médico Residente, Hospital Universitario 12 De Octubre. Área 11 Salud mental, Madrid, España. ${ }^{b}$ Médico Residente, Hospital Universitario 12 De Octubre. Área 11 Salud mental, Madrid, España. ${ }^{c}$ Médico Adjunto, Hospital Universitario 12 De Octubre. Área 11 Salud mental, Madrid, España.

Correspondencia: Ernesto José Verdura Vizcaíno (ernestoverdura@hotmail.com)

Recibido: 25/05/2011; aceptado con modificaciones: 04/02/2012

RESUMEN: Introducción: El estudio de la concentración de psicofármaco en sangre o en suero permite individualizar el perfil farmacocinético del sujeto tratado. Esto se conoce como monitorización de fármacos. Actualmente se pueden analizar antidepresivos, antipsicóticos, estabilizadores del ánimo, fármacos sustitutivos de los opioides, ansiolíticos e hipnóticos.

Objetivos: Revisar el estado actual del conocimiento sobre monitorización de antipsicóticos, de utilidad tanto para evaluar la adherencia terapéutica como para conocer la concentración del fármaco en plasma.

Metodología: Se revisaron los trabajos publicados sobre monitorización de antipsicóticos desde 1975 hasta 2010. Como fuente de información se empleó: Embase, Medline y Pubmed.

Conclusiones: La monitorización de antipsicóticos, así como de otros psicofármacos, es una práctica establecida que debe conocerse y ser objeto de actualización por parte del psiquiatra interesado en un ejercicio terapéutico basado en datos objetivos.

PALABRAS CLAVE: niveles, monitorización, psicofármaco, antipsicóticos.
ABSTRACT: Introduction: Analysis of psychotropic drug concentration in blood or serum is a mean to ascertain the pharmacokinetic profile of the treated subject. This is known as drug monitoring. Currently plasma antidepressants, antipsychotics, mood stabilizers, drugs substitutes for opioids, anxiolytics and hypnotics can be monitorized.

Methodology: We reviewed all published articles on monitoring of psychotropic drugs in general from 1975 to 2010 . As a source of information used: Embase, Medline and Pubmed.

Objetive: To review the current state of science in relation to the monitoring of atypical antipsychotics. Antipsychotic plasma level monitoring is crucial both to assess adherence and to determine the plasma drug concentration.

Conclusions: The monitoring of antipsychotics and other psychotropic drugs, is an established practice must be known and be maintained by a psychiatrist, like other aspects of psychopharmacology, for a therapeutic exercise based on the current state of science.

KEY WORDS: concentration, monitorization, psychotropic drug, antipsychotic.

\section{Introducción}

\section{Concepto}

El primer método para monitorizar antidepresivos se introdujo en los años 60. Desde entonces el número de fármacos cuya concentración puede ser evaluada es cada vez mayor. 
Los fármacos cuyos niveles se pueden medir actualmente son: antidepresivos, antipsicóticos, estabilizadores del ánimo, fármacos sustitutivos opioides, ansiolíticos e hipnóticos. En este trabajo nos centraremos en los datos actuales sobre monitorización de antipsicóticos.

Existen escasas referencias bibliográficas que indiquen como se debe realizar la monitorización y que de forma específica determinen cuales son los valores de referencia a tener en cuenta cuando se lleva a cabo dicha medición de la concentración en plasma del fármaco. A excepción de la TASK Force on The Use of Laboratory Test in Psychiatry (1985) (1) así como un Update de Orsulak (1989) (2), las guías de consenso para monitorización terapéutica de fármacos en psiquiatría aún no se han publicado (3).

Como referencias puntuales, Linder y Keck destacan por sus trabajos en relación con la monitorización de antidepresivos tricíclicos y Laux y Riederer en 1992 publicaron un artículo sobre el estado actual de la monitorización de fármacos (4, $5)$.

Como grupo destacamos, un conjunto de expertos interdisciplinario, el AGNP (Arbeitsgemeinschaft für Neuropsycopharmacologye und PharmaKopsychiatrie) formado por químicos, bioquímicos, farmacólogos y psiquiatras, que revisan la información existente en la literatura tratando de elaborar guías que ayuden a psiquiatras y personal de laboratorio que trabaja en psicofarmacología para optimizar el uso de la monitorización de psicofármacos. Incluso se dan recomendaciones para combinar el uso de la monitorización plasmática de psicofármacos con procedimientos de genotipaje/fenotipaje en farmacopsiquiatría (3).

En los últimos años se está combinando la monitorización de psicofármacos en plasma con los estudios por imagen, en concreto con la técnica de PET para medir el porcentaje de ocupación de receptores por el fármaco psicoactivo, valorando la correlación existente entre concentración de fármaco en plasma, porcentaje de ocupación de receptores y eficacia clínica.

A las técnicas anteriores se añaden de manera progresiva y complementaria, otras como son el genotipaje y el fenotipaje del paciente, reservadas actualmente solo a determinados casos y realizadas en un número de centros todavía muy reducido. Sin embargo se considera que dichas técnicas protagonizarán el futuro de la monitorización de fármacos. El fenotipaje, en el contexto de la monitorización de psicofármacos y más en concreto en relación con su farmacocinética, se refiere al estudio del funcionamiento de los enzimas responsables del metabolismo del fármaco. Mientras que el genotipaje se refiere al estudio de las variantes alélicas presentes en el individuo, cuya expresión son los diferentes enzimas responsables del metabolismo; dicho estudio tiene un resultado más estable en el tiempo, además de estar menos condicionado por el ambiente (el estudio directo de los enzimas puede dar resultados engañosos ya que su actividad puede estar condicionada por la presencia en el ambiente de sustancias inductoras o bien inhibidoras). 


\section{Consideraciones prácticas generales}

Desde un punto de vista práctico es importante considerar una serie de aspectos antes de solicitar la monitorización de un fármaco en plasma.

Lo primero que se debe tener en cuenta es, lógicamente, la presencia o no en el medio terapéutico en el que se desarrolle la actividad, de un método disponible para medir el fármaco en cuestión.

El segundo aspecto relevante es la consideración del momento en el que debemos solicitar la recogida de la muestra, que ha de hacerse desde dos perspectivas. La primera se refiere al momento en el que se debe solicitar la muestra desde que el fármaco ha sido introducido como tratamiento nuevo en el paciente o bien desde que se ha modificado su dosificación. Este aspecto es muy importante ya que se considera que la concentración del fármaco es estable cuando ha pasado un tiempo equivalente a $4-5$ vidas medias desde que se introdujo $(3,7)$.

Por tanto este es el tiempo que se considera prudente esperar para solicitar la muestra del fármaco tras su introducción o modificación, asegurando que el resultado corresponda a la concentración estable del mismo.

La segunda perspectiva a considerar, es el momento en que se debe tomar la muestra desde la última dosis. En general, para evitar que la muestra coincida con momentos en los cuales la concentración del fármaco en plasma se encuentra en su pico máximo o bien es la mínima, se recomienda que la recogida se realice entre 11 y 13 horas tras la última dosis. En el caso de fármacos en formulación depot, se considera que la toma de la muestra debe realizarse inmediatamente antes de la inyección del fármaco que corresponda, es decir al final del periodo inter-dosificación.

Otro aspecto que es imprescindible valorar antes de solicitar niveles de un fármaco es la existencia de un rango terapéutico definido para dicho fármaco, así como el nivel de evidencia con el que dicho rango se ha establecido. Esto es fundamental ya que de ello depende el valor clínico que se otorgue a la hora de interpretar una concentración como subterapéutica (aquella que no alcanza la concentración mínima eficaz), como terapéutica (aquella que se encuentra entre la concentración mínima eficaz y la concentración máxima segura o sin efectos adversos) y a un resultado compatible con una concentración supraterapéutica, que sería aquella que supera el intervalo de concentración del fármaco que siendo eficaz se considera seguro $(6,7)$.

Además de considerar la existencia o no de un rango terapéutico, se deben tener en cuenta aquellos casos en los que la monitorización se realiza exclusivamente para valorar la adherencia terapéutica o para justificar la etiología de una intoxicación.

También se dan situaciones, si bien menos frecuentes pero no por ello menos importantes, en las que la eficacia de un fármaco es difícil de comprobar mediante 
la observación clínica, teniendo que recurrir a la obtención de niveles y basando la confianza en su eficacia en el hecho de que la concentración sea terapéutica.

También se realizará en aquellos casos en los que se sospeche la existencia de una posible interacción con otro fármaco. Dicha interacción puede condicionar niveles superiores o inferiores al nivel óptimo $(6,7)$.

Para la monitorización de psicofármacos, se analizan muestras biológicas, principalmente mediante Cromatografia Líquida de alta definición, (HPLC), así como otros procedimientos en ocasiones combinados con lo anterior, tales como electroforesis capilar y cromatografía por gas (8).

\section{Niveles de recomendación de monitorización}

Existen cinco niveles de recomendación, desde el nivel uno que sería aquel que se aplica a fármacos en los que la indicación de la monitorización es elevada en base a la evidencia científica, hasta el nivel cinco aplicable a aquellos fármacos en los que no es recomendable su monitorización (3).

A continuación se exponen los diferentes niveles de recomendación.

El nivel uno sería aquel en el que la monitorización está claramente recomendada, debido a que el nivel de evidencia se basa en ensayos clínicos que muestran el beneficio de esta. En este nivel, una concentración supraterapéutica se debería considerar de riesgo de efecto adversos, una concentración subterapéutica como aquella cuyo resultado lo más probables es que sea similar a placebo y una concentración terapéutica como óptima.

En el nivel dos se encontrarían aquellos fármacos cuya monitorización simplemente está recomendada. En la literatura existe al menos un estudio prospectivo bien diseñado con criterios de objetivos definidos, así como artículos de efectos adversos a dosis supraterapéuticas. En este nivel el riesgo de una concentración por encima del rango en cuanto a efectos adversos o por debajo en cuanto a ausencia de respuesta, es menor.

En el nivel tres se clasifican los fármacos cuya monitorización se considera útil. Dicha recomendación viene respaldada por estudios retrospectivos y casos publicados sin que exista una experiencia sistemática. Una vez obtenidos los resultados solo se aspira a modificar la dosificación valorando la correspondencia entre dosis y nivel plasmático así como optimizar la respuesta en pacientes cuya respuesta es débil.

Los fármacos que corresponden al nivel cuatro se caracterizan porque su monitorización podría ser útil, basada en datos clínicos consistentes. Sería empleada para controlar si las concentraciones plasmáticas son proporcionales a la dosis administrada.

El nivel cinco se reserva para fármacos cuya monitorización no está recomendada, ya que su evidencia se basa exclusivamente en libros de texto y farmacología básica (3). 
ORIGINALES Y REVISIONES

\section{Metodología}

Se realizó una exhaustiva revisión bibliográfica sobre la evidencia encontrada hasta el momento en cuanto a la monitorización de psicofármacos. Tabla 1. Dicha revisión se estructuró en tres pasos. Inicialmente se revisaron todos los artículos publicados sobre la monitorización de psicofármacos en general desde 1950 hasta 2010. Posteriormente se seleccionaron los trabajos sobre monitorización de antipsicóticos, publicados en el mismo intervalo de tiempo, primándose los trabajos originales y metaanálisis independientes de la industria. Finalmente se revisaron las referencias bibliográficas que aparecían en los artículos y no habían sido revisadas. Como fuente de información se empleó Embase, Medline y Pubmed.

Tabla 1.

Se muestran las referencias bibliográficas de cada antipsicótico en relación con el tipo de estudio realizado.

\begin{tabular}{|c|c|c|c|c|c|c|c|c|c|}
\hline & Amisulprida & Aripiprazol & Clozapina & Olanzapina & Quetiapina & Risperidona & $\begin{array}{l}\text { Risp. Liber. } \\
\text { prolongada }\end{array}$ & Paliperidona & Ziprasidona \\
\hline $\begin{array}{l}\text { Ensayos } \\
\text { clínicos }\end{array}$ & 15 & & 30 & 37 & 44 & $60,69,70$ & 49 & $61,62,69$ & 73 \\
\hline Revisión & 14,45 & $8,45,66$ & $\begin{array}{l}20,23,24,26 \\
27,31,33,45\end{array}$ & $8,27,45$ & $8,27,43,45$ & $8,27,45$ & 54 & & 27,45 \\
\hline \begin{tabular}{|l|} 
Est. \\
prospectivo
\end{tabular} & $11,12,16$ & 18,19 & \begin{tabular}{|}
$22,29,34,35$ \\
$36,32,64,71$
\end{tabular} & $\begin{array}{c}32,38,39 \\
40,41\end{array}$ & & $\begin{array}{c}51,52,53, \\
55,56,\end{array}$ & 57,68 & & 72 \\
\hline \begin{tabular}{|l|} 
Est. \\
retrospectivos
\end{tabular} & & & 25,28 & 42 & 46 & 50 & & 63 & 74 \\
\hline \begin{tabular}{|l|} 
Casos \\
publicados
\end{tabular} & & & 36 & & & & & & \\
\hline \begin{tabular}{|l|}
$\begin{array}{l}\text { Datos clínicos } \\
\text { consistentes }\end{array}$ \\
\end{tabular} & & & & & & & 48,59 & & \\
\hline $\begin{array}{l}\text { Libros texto y } \\
\text { farmacología } \\
\text { básica }\end{array}$ & 7 & 7 & 7 & 7 & & 7 & & & \\
\hline \begin{tabular}{|l|} 
NIVEL RECO- \\
MENDACIÓN \\
MONITORI- \\
ZACIÓN \\
\end{tabular} & 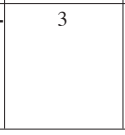 & & 1 & 1 & 3 & 2 & & 2 & 4 \\
\hline
\end{tabular}

\section{Resultados}

\section{Monitorización de antipsicóticos}

La monitorización de antipsicóticos es fundamental, tanto para determinar la adherencia terapéutica de los pacientes como para conocer de manera individualizada la concentración de fármaco en plasma, la cual tanto por la variabilidad genética en su metabolismo como por la interacción con otros psicofármacos, condiciona una elevada variabilidad en la farmacocinética interindividual (9). 
La utilidad de su monitorización está bien determinada para antipsicóticos clásicos tales como haloperidol, perfenacina y flufenazina.

Para los antipsicóticos atípicos existe una menor experiencia. La monitorización de clozapina, olanzapina y risperidona es una práctica bien establecida (3), sin embargo para los demás los resultados no tienen una evidencia tan amplia; aunque si se defiende su práctica para mejorar la eficacia sobre todo en pacientes que no responden a dosis terapéuticas o que desarrollan efectos adversos (10).

A su vez se puede establecer la correlación entre concentración en plasma del antipsicótico y la ocupación de receptores D2 obtenida mediante la tomografía por emisión de positrones; dichos estudios proporcionan información adicional a la monitorización de la concentración. Se trata de una técnica puramente experimental, siendo además compleja su interpretación puesto que se debe tener en cuenta la naturaleza del radioligando (10).

Se presenta a continuación un resumen del estado actual de la ciencia en cuanto a la monitorización de antipsicóticos atípicos.

\section{Amisulprida}

La amisulprida es un antipsicótico atípico que debido a su perfil farmacodinámico único puede ser útil para pacientes mayores con esquizofrenia $(11,12)$. Se ha demostrado como en mayores de 60 años se logra eficacia terapéutica a menores concentraciones, lo cual se debe tener en cuenta a la hora de establecer su dosificación (11). Ni la disminución de su aclaramiento con la edad ni la comedicación y comorbilidad presente en pacientes mayores de 60 años, son factores que contraindiquen su uso en población de edad avanzada $(12,13)$.

La monitorización de la amisulprida ha demostrado capacidad predictiva para no respondedores y para la aparición de síntomas extrapiramidales. Sin embargo los análisis estadísticos realizados demuestran que para que un paciente se beneficie de la monitorización de dicho fármaco en lo que se refiere a mejoría de respuesta y una disminución de síntomas extrapiramidales, se debe monitorizar al menos a 7 pacientes. Es decir, de cada 7 pacientes con escasa respuesta solo en 1 los niveles serán subterapéuticos y su normalización permitirá mejorar la eficacia y de cada 7 pacientes con síntomas extrapiramidales, solo en 1 de ellos los niveles serán supraterapéuticos y su disminución permitirá mejorar dichos efectos adver$\operatorname{sos}(9,10)$.

Su concentración en plasma se correlaciona con la dosis, ocupación dopaminérgica, respuesta y síntomas extrapiramidales. La respuesta clínica óptima se encuentra a dosis de 400-800 mg/día, para esquizofrenia y trastorno esquizoafectivo, la cual se corresponde a niveles plasmáticos de $200-500 \mathrm{ng} / \mathrm{ml}$, con una ocupación dopaminérgica de al menos el 65\% a nivel estriatal y extraestriatal (14). 
Se ha sugerido la posible presencia de efectos adversos con niveles de 336 $\mathrm{ng} / \mathrm{mL}, 377 \mathrm{ng} / \mathrm{mL}$ y $395 \mathrm{ng} / \mathrm{mL}$. Un nivel plasmático por debajo de $320 \mathrm{ng} / \mathrm{mL}$ parece predecir ausencia de efectos adversos (11). Los efectos extrapiramidales aparecen a partir de $320 \mathrm{ng} / \mathrm{ml}$, así como la ocupación de receptores dopaminérgicos de al menos el $78 \%$. La ausencia de efecto terapéutico se encuentra probablemente por debajo de $200 \mathrm{ng} / \mathrm{ml}$, correspondiendo a una dosis de aproximadamente 400 mgr/d y un porcentaje de ocupación del $65 \%$ (14).

Se sugiere por tanto un rango entre $200 \mathrm{ng} / \mathrm{mL}$ y $320 \mathrm{ng} / \mathrm{mL}$ para una respuesta óptima sin efectos adversos, pero no se ha sistematizado en la clínica, quizá debido a diversas dificultades como la relación variable que existe entre niveles plasmáticos y dosis, y en menor medida por la variación en los niveles presente en mujeres y ancianos, ligeramente superior en ambos, probablemente por diferencias en la función renal, peso y volumen de distribución (13-15), aunque con escasa repercusión en la eficacia o en los efectos adversos $(7,10)$.

La comedicación con litio y clozapina parece aumentar los niveles del fármaco, mientras que otras como las benzodiacepinas y otros antipsicóticos no lo modifican.

También se ha observado que el consumo de tabaco parece no tener correlación con la concentración de amisulprida, a diferencia de lo que ocurre con otros antipsicóticos (16).

Se ha demostrado que la relación entre dosis y respuesta es elevada, además los efectos adversos se controlan bien modificando la dosis, lo cual simplifica su manejo. Por ello la monitorización se reserva a casos en los que la respuesta clínica es escasa, se cuestiona la adherencia, o en casos en los que la presencia de otras drogas o enfermedades pueden aumentar el riesgo de efectos adversos.

\section{Aripiprazol}

La evidencia actual defiende que una dosis de 10mgr/día de aripiprazol es eficaz en esquizofrenia y trastorno esquizoafectivo, utilizando dosis aproximadas de $10 \mathrm{mg}$ /día se debe obtener una respuesta clínica mantenida y una ocupación de receptores D2 adecuada $(17,18)$.

En cuanto a su farmacocinética, lo más relevante en relación a dicho fármaco, es su metabolismo por el CYP 3A4 y por el CYP 2D6, dando lugar principalmente a su metabolito dehydroaripiprazol (18). La variabilidad de la molécula activa aripiprazol más dehydroaripiprazol es un 15-30\% menor que de aripiprazol solo, es decir la mayor variabilidad es debida a su metabolismo, una vez metabolizado el conjunto de ambas moléculas es poco variable de forma interindividual (19).

Se ha encontrado mejoría en sujetos en los que se alcanzan niveles en suero entre 150 y $300 \mathrm{ng} / \mathrm{ml}$. Los efectos adversos parecen ser nulos entre 110 y $210 \mathrm{ng} /$ 
$\mathrm{ml}$ y ligeros entre 210 y $249 \mathrm{ng} / \mathrm{ml}$ (7). Se consideran niveles entre $150 \mathrm{ng} / \mathrm{mL}$ y $210 \mathrm{ng} / \mathrm{mL}$ como adecuados para obtener efectos beneficiosos sin la presencia de efectos adversos extrapiramidales.

Género, edad, genotipo de los enzimas metabolizadores y comedicación influye en su variabilidad, sin embargo la relevancia clínica de esta variabilidad es mínima por lo que no justifica su monitorización (7).

\section{Clozapina}

La clozapina resulta eficaz en el 50-60\% de los pacientes con esquizofrenia resistentes a otros antipsicóticos, como indica el estudio de Meltzer y colaboradores. Su uso ha quedado restringido a casos resistentes a otros neurolépticos por el riesgo de provocar agranulocitosis, haciendo necesaria habitualmente su monitorización (20-21).

En cuanto a su farmacocinética, la clozapina es metabolizada a nivel hepático por diferentes isoenzimas del citocromo p 450, en diferentes productos de la biotransformación. Jann y colaboradores, destacan dentro de sus diferentes metabolitos, en base a su nivel de actividad y cuantía, a la forma desmetilada, norclozapina. La disponibilidad de la clozapina se ve influida por factores tales como la edad, género, hábito tabáquico (recordar que el tabaco es inductor del CYP1A2), peso, ingesta de cafeína y por la ingesta concomitante de otras sustancias (23-25).

Estos factores mencionados influyen en su disponibilidad, lo cual explica la gran variabilidad interindividual que presenta la concentración de clozapina en plasma. Sirva a modo de ejemplo, la diferencia en la concentración plasmática de clozapina en función del género, encontrándose en la mujer una concentración significativamente mayor de la que presenta en el varón; este hecho se debe en parte a que en el organismo femenino, a igualdad de dosis $/ \mathrm{kg}$, la menor actividad del citocromo CYP1A2 condiciona un menor aclaramiento del fármaco. Además de la presencia de un metabolismo lentificado, la mayor proporción de grasa en el cuerpo femenino, implica un mayor volumen de distribución y por tanto, una vida media más larga (3).

Debido a la variabilidad farmacocinética mencionada, Freeman y Oyewumi en 1997, sugieren que la monitorización de las concentraciones de clozapina en plasma podría ser útil en el manejo clínico $(26,27)$.

Los estudios con clozapina muestran una relación positiva entre concentración en plasma y respuesta terapéutica, con un margen asociado a buena respuesta clínica entre 350 y $420 \mathrm{ng} / \mathrm{ml}$ (22). Devinski, Olesen y Van der Zwaag afirman que la incidencia de confusión, mareos, convulsiones y otros efectos adversos también aumenta a medida que aumenta la concentración del fármaco (28-30). Debido a la relación positiva entre concentración en plasma de clozapina y tanto respuesta 
ORIGINALES Y REVISIONES

terapéutica como efectos adversos, surge controversia sobre dónde situar el umbral terapéutico de clozapina. Para Olesen situar dicho umbral por encima de $400 \mathrm{ng} / \mathrm{ml}$ requeriría administrar dosis innecesariamente altas en muchos pacientes quedando expuestos a importantes efectos adversos considerándose un nivel innecesario. Sin embargo dicho autor opina que un umbral más bajo, alrededor de $250 \mathrm{ng} / \mathrm{ml}$ podría proporcionar una guía más útil para el ajuste de clozapina.

Otra posibilidad sería establecer como punto de corte una concentración en plasma de $350 \mathrm{ng} / \mathrm{ml}$ la cual logra distinguir entre respondedores y no respondedores, teniendo en cuenta que por debajo de este nivel un elevado porcentaje de sujetos también responden.

Esto lleva a Olesen a la afirmación de que un porcentaje elevado de los sujetos en tratamiento con clozapina, obtienen un efecto terapéutico con una concentración por debajo de este valor, lo cual hace que no deba considerarse como umbral terapéutico, sino como un nivel cuyo valor es deseable alcanzar en pacientes que inicialmente no responden. Además debido a la mala tolerancia que tiene el ascenso rápido de la dosis de clozapina, así como a la posible estabilización de algunos pacientes con bajas dosis (y bajos niveles), los cuales desarrollan una respuesta positiva después de algunas semanas, el intentar alcanzar un nivel en inicio por encima de $350 \mathrm{ng} / \mathrm{ml}$ podría generar una toxicidad innecesaria. Conocer las concentraciones de clozapina en plasma podría proporcionar una guía de manejo útil en pacientes que no responden al ajuste empírico de la dosis estándar, o en aquellos que pueden estar consumiendo sustancias que interfieran potencialmente en el metabolismo de clozapina (31). Por consiguiente, los datos que existen en la actualidad permiten sugerir un nivel de concentración de clozapina en plasma de $200 \mathrm{ng} / \mathrm{ml}$ como suficiente para evitar la recaída $(31,32)$. Incrementar la dosis para alcanzar niveles en plasma de 350-400 ng/ml podría estar especialmente indicado en pacientes sin efectos adversos los cuales no presentan mejoría psicopatológica a dosis estándar o a bajas concentraciones (31).

El enunciado anterior se ve respaldado por los estudios de neuroimagen realizados con tomografía por emisión de positrones, los cuales demuestran que la máxima ocupación de receptores se alcanza a concentraciones de clozapina en plasma entre 200 y $400 \mathrm{ng} / \mathrm{ml}$. En el caso de la clozapina, las concentraciones plasmáticas del fármaco se correlacionan bien con la ocupación de receptores D4 pero no tan bien con la ocupación de receptores D2 o D3; lo cual es coherente con la relevancia del bloqueo D4 para la clozapina en comparación con el bloqueo D2, efecto principal de los otros antipsicóticos, sobre todo los clásicos (33).

En relación con la interacción de la clozapina con otros fármacos, es necesario considerar de nuevo su metabolismo, ya que es en esta fase de su farmacocinética en la cual es más vulnerable a la presencia de otros fármacos. El primer enzi- 
ma responsable de convertir clozapina en norclozapina es el citocromo CYP1A2 mientras que el CYP3A4 es el primer enzima involucrado en el paso de clozapina a clozapina-N-oxido $(33,34)$.

La fluvoxamina y la cimetidina debido a la inhibición que ejercen sobre los enzimas mencionados previamente, pueden disparar la concentración de clozapina (33-35). Por ejemplo, como afirma Hiemke, si se añaden $100 \mathrm{mg}$ de fluvoxamina a un paciente que toma $150 \mathrm{mg}$ de clozapina la concentración pasará de $294 \mathrm{ng} / \mathrm{ml}$ a $2470 \mathrm{ng} / \mathrm{ml}(35)$.

Además de los fármacos habituales cuya interacción con clozapina es conocida, se están investigando otros fármacos de uso habitual que pueden tener repercusión importante a nivel del metabolismo de la clozapina. Cabe destarcar la interacción existente con el ciprofloxacino; posiblemente la inhibición del CYP1A2 y CYP3A4 retrasa el metabolismo de la clozapina y eleva su concentración plasmática, pudiendo ocasionar efectos adversos. Por ello se recomienda utilizar otro antimicrobiano o bien reducir la dosis de clozapina y monitorizar su concentración mientras se utiliza en combinación con ciprofloxacino (36).

Hasta el momento no se ha establecido un valor umbral como techo de los niveles de concentración de clozapina. Los niveles elevados parecen predecir cambios electroencefalográficos y las convulsiones suelen ocurrir en pacientes con una concentración de clozapina en plasma en torno a 1000microgr/L. por lo que dicha concentración debe mantenerse en un rango inferior (7).

Resulta prudente emplear medidas profilácticas anticomiciales y de las mioclonías cuando los niveles plasmáticos de clozapina llegan a $500-600 \mathrm{ng} / \mathrm{mL}$ y obligado cuando estos alcanzan cifras de $1000 \mathrm{ng} / \mathrm{mL}$ (7).

\section{Olanzapina}

La concentración en plasma de Olanzapina se ve influida por muchos factores: el género, siendo mayor la concentración en mujeres que en hombres, el hábito tabáquico (inductor del CYP1A2), el cual disminuye la concentración, la edad, en relación a la cual se observa que el ratio concentración/dosis se incrementa en un $9,4 \%$ con cada década que avanza el sujeto y la comedicación con fármacos inhibidores enzimáticos los cuales también elevan la concentración de olanzapina (38-40).

Los estudios con olanzapina demuestran una fuerte asociación entre niveles de concentración en plasma y beneficio clínico. Su monitorización ha demostrado ser útil tanto para controlar su eficacia como para valorar el riesgo de efectos adversos $(41,42)$.

Existe consenso en afirmar que niveles superiores a $40 \mathrm{ng} / \mathrm{ml}$ no proporcionan beneficio terapéutico (40). La toxicidad severa es poco común, se presenta cuando la concentración del fármaco supera los $100 \mathrm{ng} / \mathrm{mL}$ y ocasionalmente se 
ORIGINALES Y REVISIONES

han dado casos de muerte con niveles por encima de $160 \mathrm{ng} / \mathrm{mL}$ (generalmente cuando otras drogas o factores físicos se ven implicados) $(41,42)$.

Un rango adecuado de concentración plasmática para olanzapina, sin la presencia de efectos adversos y con una adecuada respuesta terapéutica se sitúa entre los 20 y $40 \mathrm{ng} / \mathrm{mL}$, (12h tras la dosis), para pacientes con esquizofrenia y trastorno bipolar en fase de manía $(7,42)$.

En la práctica la dosis se suele manejar en función de la respuesta y tolerabilidad, sin el empleo de la monitorización del fármaco de manera rutinaria. Las determinaciones en plasma suelen reservarse para aquellos en los que se sospecha mala adherencia o para los que no responden a dosis máximas (7).

\section{Quetiapina}

La quetiapina es una dibenzodiazepina utilizada en nuestro medio principalmente para el tratamiento de síntomas psicóticos, aunque sus indicaciones están aumentando en los últimos años. De los 11 metabolitos encontrados tras su metabolismo hepático, dos de ellos son activos. Parece que su concentración en plasma oscila entre el 2 y el $12 \%$ respecto de la molécula de quetiapina, dando la impresión de que su papel como agentes activos es muy escaso (43).

Los datos sugieren que el tabaco no influye en su metabolismo. A su vez, quetiapina a concentraciones clínicas no parece tener efecto sobre CYP1A2, 2C9, 2C19, 2D6. Existen dudas sobre su posible interacción a nivel de CYP3A4 tras un reciente estudio en el que demuestra la interacción entre armodafinilo (inductor CYP3A4) y quetiapina adeministrados de forma conjunta (44). No tiene efecto en las concentraciones séricas de Litio, a dosis terapéuticas lo que facilita la comedicación (43).

Se ha demostrado como la fenitoína y la tioridacina, incrementan su eliminación, mientras que el ketoconazol, la disminuye. Con ellos, así como con otros potentes inductores o inhibidores de CYP $3 \mathrm{~A} 4$, se deben realizar ajustes de dosis $(45,46)$.

Debido al potente efecto inductor del metabolismo de quetiapina por la carbamazepina se recomienda no utilizar ambos medicamentos simultáneamente (46).

Por otro lado, se recomienda disminuir la dosis en ancianos y en pacientes en los que se administra simultáneamente clozapina o fluvoxamina (46).

En los estudios realizados con quetiapina, no se ha encontrado significación estadística en la correlación entre concentraciones plasmáticas de quetiapina y su respuesta clínica medida según las medidas habituales de la severidad de los síntomas psicóticos (43-46).

Además y de manera similar, los estudios de imagen con tomografía por emisión de positrones muestran discrepancia entre el tiempo durante el cual el receptor se encuentra ocupado y las concentraciones de quetiapina en plasma (17). Por todo 
lo referido existe cierta controversia en cuanto a la utilidad de monitorizar los niveles de quetiapina en plasma. No obstante, se han propuesto diversos valores como posible nivel umbral, por ejemplo en $77 \mathrm{microgr} / \mathrm{L}$ (45) y entre 50-100 microgr/L (47).

\section{Risperidona}

Los efectos farmacológicos de la risperidona, dependen de la suma de la concentración en plasma de risperidona y de su metabolito 9-hidroxirisperidona (principio activo de la paliperidona), por lo que se deben monitorizar ambas moléculas, para evitar los errores derivados de la monitorización de una de ellas de forma aislada $(15,45)$.

La actividad del metabolito 9-hidroxirisperidona es nueve veces menor que la actividad de la molécula de la cual deriva, siendo su perfil farmacológico similar (48).

Además y quizá como factor implicado en la afirmación previa, existen estudios en animales que sugieren que la risperidona atraviesa mejor la barreara hematoencefálica que su metabolito 9-hidroxirisperidona (49).

Por otro lado, se debe considerar que el $90 \%$ de la molécula activa está formada por 9-hidroxirisperidona (50).

En cuanto a la dosificación de este antipsicótico atípico, la literatura defiende que $6 \mathrm{mgr} / \mathrm{d}$ es una dosis óptima para pacientes con esquizofrenia (50-52). Según lo revisado en diferentes estudios, entre los que destacan el de Aveosos y el de Spina E se puede concluir la existencia de cierta correlación lineal entre la dosificación del fármaco y la concentración de la molécula activa, aunque esta es una correlación débil $(53,54)$.

En cuanto a la utilidad clínica del conocimiento de dichos niveles, se ha observado que en pacientes con esquizofrenia crónica que experimentan una reactivación, los niveles en plasma de risperidona y de su metabolito activo se correlacionan con la aparición de síntomas de parkinsonismo; sin embargo no existe una correlación significativa con la mejoría clínica. Tampoco se ha encontrado correlación entre dosis de risperidona y efectos anticolinérgicos, pero sí entre la dosificación y los efectos extrapiramidales (48). Es decir, tanto la concentración como la dosificación presentan correlación con los efectos adversos extrapiramidales de la risperidona, siendo escasa la evidencia de tal correlación para el efecto terapéutico.

Parece que el género no es un factor que influya en la concentración del fármaco, la cual sí que se muestra influida por la edad (48).

Otro aspecto a considerar es la utilidad de la monitorización en la fase de transición entre la forma oral y la formulación depot $(51,55,56)$. Según indicación farmaceútica la dosis recomendada de risperidona depot son $50 \mathrm{mgr}$ cada dos se- 
ORIGINALES Y REVISIONES

manas; los ensayos clínicos revisados vienen a decir que una dosis de 75 mgr cada dos semanas produce efectos adversos sin beneficio clínico (55). En cuanto a la transición entre la formulación oral y la intramuscular, se recomienda en pacientes estables comenzar con dosis de $25 \mathrm{mgr}$ en la forma intramuscular cada dos semanas mientras que se mantiene el tratamiento de risperidona oral entre 2 y 6 mgr, durante al menos las dos primeras dosificaciones intramusculares; se postula que $25 \mathrm{mgr}$ de la forma inyectable equivalen a $2 \mathrm{mgr}$ de la formulación oral $(48,55,56)$.

Los niveles plasmáticos obtenidos con la formulación depot, realmente se estabilizan tras las seis semanas de tratamiento, siendo posteriormente las oscilaciones en plasma, menores que las presentadas por la formulación oral. Incrementos en la dosis aumentan los niveles así como disminuyendo esta, se reduce la concentración, aunque estos efectos no se aprecian hasta transcurridas entre 3 y 4 semanas tras el cambio de dosis.

La medición de niveles en la transición de la formulación oral a la formulación depot, así como durante los cambios de dosificación en esta última, puede permitir a los clínicos anticipar los posibles resultados de variación de dosis y entender las recomendaciones de manejo $(10,55-58)$.

Se plantea que las diferencias interindividuales de la isoforma CYP 2D6, la cual es responsable de una parte significativa del metabolismo de la risperidona, además del citocromo $\mathrm{P} 4503 \mathrm{~A}$, puedan influir en los niveles del fármaco en plasma y en la respuesta al mismo (52). Se ha visto que dichos polimorfismos aunque no alteran la eficacia clínica, si que afectan a los ratios de risperidona y 9-hidroxirisperidona, confirmándose una de las dos hipótesis iniciales $(51,52)$. Por ello se debe considerar que para pacientes que metabolizan la risperidona lentamente una dosis media puede resultar en niveles plasmáticos excesivos, los cuales pueden provocar efectos adversos por lo que una posibilidad sería iniciar en ellos el tratamiento directamente con paliperidona (9-hidroxirisperidona) (51). Otro aspecto que se ha observado en la monitorización de risperidona inyectable de acción prolongada es que en aquellos pacientes en los que es eficaz, se encuentran mayores concentraciones de 9-hidroxirisperidona que de risperidona. Sin embargo el significado de este hallazgo no se ha determinado (57).

En resumen, la monitorización de risperidona puede ser útil en pacientes con baja respuesta clínica y tolerancia por la posible dificultad a la hora de determinar la dosis individual oral del fármaco así como para lograr una dosificación óptima en base a la titulación $(7,47)$. La monitorización de risperidona inyectable de acción prolongada también es útil, principalmente en casos de baja tolerancia al fármaco (57).

Aunque no existe un rango terapéutico de concentración en plasma claramente definido para risperidona, se puede establecer para el manejo un rango tera- 
péutico que oscila entre $20-60 \mathrm{ng} / \mathrm{mL}$ de molécula activa (risperidona más 9-OHrisperidona).

60). Estos niveles suelen alcanzarse con dosis orales entre 3 y $6 \mathrm{mg} / \mathrm{d}(7,50,57-$ podría situarse en $74 \mathrm{ng} / \mathrm{ml}$ (54). A partir de $60 \mathrm{ng} / \mathrm{ml}$ la ocupación de receptores D2 es del 84\%, umbral de ocupación que se asocia con aparición de SEP (55).

\section{Paliperidona}

La paliperidona es un antipsicótico atípico. Es idéntico al metabolito activo de la risperidona, la 9- $\mathrm{OH}$ - risperidona, siendo su funcionamiento similar a la risperidona, mostrando afinidad sobre el receptor D2 y 5HT2 $(61,62)$. La formulación de liberación prolongada permite una menor cantidad de picos y valles, lo cual aumenta la tolerabilidad y puede explicar la posibilidad de emplear dosis recomendadas de 6-9 mgr/d, mayores que de risperidona (4-6 mgr/d).

El rango de concentración plasmática recomendado se sitúa en torno a 20-52 $\mathrm{ng} / \mathrm{ml}$, muy similar al de risperidona, 20-60 ng/ml (63).

Concentraciones de paliperidona de 16 a $27 \mathrm{ng} / \mathrm{ml}$, bastante por debajo de lo anterior, conllevan una ocupación de receptores D2 a nivel estriatal del 65-80\%, considerada óptima para el tratamiento antipsicótico (64-66). Para risperidona la ocupación óptima se alcanzaba con niveles de 15-40 ng/ml o mayor (67-68).

A nivel metabólico es determinante la actividad de CYP 3A4 pero no de CYP 2D6 que si lo era en el metabolismo de risperidona para transformarla en 9-hidroxirisperidona, por ello la variabilidad en plasma de paliperidona será menor en relación a las alteraciones debidas a este isoenzima. La glicoproteina $\mathrm{P}$ que se expresa en la mucosa intestinal y en la BHE actuando como transportador, influye en la concentración tanto de risperidona como de paliperidona (69-70). Ambos son sustratos de dicho transportador cuya expresión se ve influida por aspectos genéticos y por el efecto inductor o inhibidor de la co-medicación. La variabilidad debida por tanto al CYP 3A4 y a la glicoproteína P explica porque la variabilidad en los niveles de risperidona y de paliperidona es similar.

En resumen, la monitorización de niveles de paliperidona es útil para optimizar el tratamiento de los pacientes con este psicofármaco. El rango de $20-60 \mathrm{ng} / \mathrm{ml}$ establecido como diana terapéutica para risperidona, también se puede establecer como niveles plasmáticos óptimos de palperidona (63).

\section{Ziprasidona}

La ziprasidona es un antipsicótico atípico cuyo metabolismo se realiza principalmente a nivel de citocromo P450 3A y el enzima aldehído oxidasa. Sus metabolitos apenas tienen relevancia farmacodinámica, por ello alteraciones a nivel del 
ORIGINALES Y REVISIONES

renal y hepático presentan escasa repercusión. Sus principales efectos adversos son naúseas, sedación y mareos. Menos frecuentes son los SEP y la ganancia de peso.

La dosis recomendada para el tratamiento de la esquizofrenia oscila entre 80 y $160 \mathrm{mgr} / \mathrm{d}$. En pacientes resistentes se pueden emplear dosis en torno a 320 $\mathrm{mgr} / \mathrm{d}$, sin elevado riesgo de efectos adversos.

Para lograr su efecto antipsicótico se debe bloquear aproximadamente el 70\% de receptores D2 lo cual se correlaciona con una concentración de al menos $50 \mathrm{ng} /$ $\mathrm{ml}$ (71). A nivel clínico se recomienda alcanzar niveles entre 50 y $130 \mathrm{ng} / \mathrm{ml}$ para alcanzar una ocupación del 70-80\% de receptores. El nivel superior de ocupación al $80 \%$ a nivel de putamen se puede alcanzar con niveles de $250 \mathrm{ng} / \mathrm{ml}(72)$.

Se ha encontrado una correlación lineal significativa entre dosis y concentración en plasma de antipsicótico. El género parece no influir en la concentración plasmática, mientras que la carbamazepina si disminuye los niveles de ziprasidona $(73,74)$.

Un factor a tener en cuenta que puede ser responsable de la variabilidad en los niveles plasmáticos de ziprasidona intraindividual, es la absorción del fármaco, siendo esta mucho mayor cuando se administra con alimentos (74).

Ni la dosis ni la concentración plasmática presenta una adecuada correlación con la eficacia clínica, encontrándose la principal correlación con el porcentaje de ocupación de receptores.

Se puede concluir que la monitorización de ziprasidona es útil principalmente ante la sospecha de mal cumplimiento terapéutico (74).

\section{Conclusiones}

A pesar de que el número de psicofármacos cuya monitorización se puede solicitar es creciente, todavía son escasas las referencias bibliográficas que orienten sobre el modo de realizar esta monitorización así como sobre los valores de referencia a tener en cuenta cuando se mide la concentración del fármaco en plasma.

Probablemente las recomendaciones más sencillas y útiles que se pueden consultar en la actualidad sean las que proporciona el AGNP (Arbeitsgemeinschaft für Neuropsycopharmacologye und PharmaKopsychiatrie), grupo formado por químicos, bioquímicos, farmacólogos y psiquiatras, que revisan la información existente en la literatura tratando de elaborar guías que ayuden a psiquiatras y personal de laboratorio que trabaja en psicofarmacología. En la práctica de la psiquiatría es fundamental conocer una serie de aspectos en relación a la monitorización de psicofármacos, desde los aspectos generales hasta los detalles concretos de los fármacos principales. 
Aspectos generales a diversos niveles como: la toma de la muestra, la necesidad de esperar el tiempo equivalente a 4-5 vidas medias del fármaco antes de solicitar la medida o el hecho de tener que esperar 11-13 horas desde la última toma para obtener la muestra; también en relación con la interpretación de los resultados, la necesidad de considerar no solo el rango terapéutico del fármaco, sino integrar variables como edad, sexo, hábito tabáquico y situación clínica; finalmente cuestiones habituales como evaluar una situación clínica compatible con intoxicación o sencillamente la necesidad de corroborar la adherencia terapéutica de un paciente.

La monitorización de antipsicóticos está bien determinada para los clásicos como haloperidol, perfenacina y flufenazina.

Para los antipsicóticos atípicos existe una menor experiencia aunque parece una práctica bien establecida para algunos como clozapina, olanzapina y risperidona.

Para el amisulprida se sugiere un rango entre $200 \mathrm{ng} / \mathrm{mL}$ y $320 \mathrm{ng} / \mathrm{mL}$ para una respuesta óptima sin efectos adversos, pero no se ha sistematizado su monitorización en la práctica clínica, salvo en aquellos casos en los que cuestiona la adherencia, o en los que la presencia de otras drogas o enfermedades pueden aumentar el riesgo de efectos adversos.

En cuanto al aripiprazol, existe dificultad en base a la variabilidad interindividual, para poder establecer un rango, además la repercusión en cuanto a los niveles de factores como edad, género o comedicación es tan baja, que no justifica su monitorización.

La clozapina es un fármaco cuya monitorización se realiza de manera habitual en la práctica clínica, debido sobre todo a sus posibles efectos adversos, así como a la presencia de un rango bien establecido. Los datos permiten sugerir un nivel de concentración de clozapina en plasma de $200 \mathrm{ng} / \mathrm{ml}$ como suficiente para evitar la recaída. Incrementar la dosis para alcanzar niveles en plasma de 350$400 \mathrm{ng} / \mathrm{ml}$ podría estar especialmente indicado en pacientes sin efectos adversos los cuales no presentan mejoría psicopatológica a dosis estándar o a bajas concentraciones. Los niveles elevados parecen predecir cambios electroencefalográficos y las convulsiones suelen ocurrir en pacientes con una concentración de clozapina en plasma en torno a $1000 \mathrm{ng} / \mathrm{mL}$. por lo que dicha concentración debe mantenerse en un rango inferior.

La dosis de olanzapina en la práctica se suele manejar en función de la respuesta y tolerabilidad, sin el empleo de la monitorización del fármaco de manera rutinaria. A pesar de esto conviene recordar que niveles superiores a $40 \mathrm{ng} / \mathrm{ml}$ no proporcionan beneficio terapéutico.

La monitorización de los niveles de quetiapina en plasma tiene una utilidad controvertida. No obstante, se han propuesto diversos valores como posible nivel umbral, por ejemplo en $77 \mathrm{ng} / \mathrm{mL}$ y entre $50-100 \mathrm{ng} / \mathrm{mL}$. 
ORIGINALES Y REVISIONES

En cuanto a la posible monitorización de la risperidona, tener en cuenta que, tanto la concentración en plasma como la dosificación presentan correlación con los efectos adversos extrapiramidales de la risperidona, siendo escasa la evidencia de tal correlación para el efecto terapéutico. La medición de niveles en plasma sobre todo es una práctica frecuente a la hora de sustituir la formulación oral por la formulación depot. Aunque no existe un rango terapéutico de concentración en plasma claramente definido para risperidona, se puede establecer para el manejo un rango terapéutico que oscila entre $20-60 \mathrm{ng} / \mathrm{mL}$ de molécula activa (risperidona más 9-OH-risperidona).

Estos niveles suelen alcanzarse con dosis orales entre 3 y $6 \mathrm{mg} / \mathrm{d}$. El nivel a partir del cual podría surgir riesgo de síntomas de parkinsonismo podría situarse en $74 \mathrm{ng} / \mathrm{ml}$ de concentración en plasma.

Para la paliperidona se puede asumir como diana el mismo rango terapéutico que para risperidona, $(20-60 \mathrm{ng} / \mathrm{ml})$.

La monitorización de ziprasidona también es útil sobre todo ante la sospecha de mal cumplimiento terapéutico, recomendando unos niveles entre 50 y $130 \mathrm{ng} /$ $\mathrm{ml}$.

La monitorización de antipsicóticos, así como de otros psicofármacos, es una práctica establecida que debe conocerse y ser objeto de actualización por parte del psiquiatra, al igual que otros aspectos de la psicofarmacología, para un ejercicio terapéutico lo más ajustado posible al estado actual de la ciencia.

BIBLIOGRAFÍA:

(1) No authors listed. Tricyclic antidepressants blood level measurements and clinical outcome: an APA Task Force report. Task Force on the Use ofLaboratory Tests in Psychiatry. Am J Psychiatry, 1985 Feb; 142: 155-62.

(2) Orsulak PJ. Therapeutic monitoring of antidepressant drugs: guidelines updated. Ther Drug Monit 1989 Sep; 11: 497-507.

(3) Baumann P, Hiemke C. The AGNPTDM Expert Group Consensus Guidelines: Therapeutic Drug Monitoring in Psychiatry. Pharmacopsychiatry 2004; 37: 243-265.

(4) Mark W Linder and Paul E. Keck, Jr. Standards of laboratory practice: antidepressant drug monitoring. Clinical Chem. 1998; 44: 1073-1084.

(5) Riederer P, Laux G. Therapeutic drug monitoring of psychotropics: report of a consensus conference. Pharmacopsychiatry. 1992; 25: 271-272.

(6) Mann K, Hiemke C, Schmidt LG, Bates DW, et al. Appropriateness of therapeutic drug monitoring for antidepressants in routine psychiatric impatient care. Ther Drug Monit. 2006; 28: 83-8.

(7) Taylor D, Paton C and Kapur S. The Maudsley Prescribing Guidelines 10Th edition. London; Informa healthcare, 2009; 1-6. 
(8) Musenga A, Saracino MA, Sani G, Raggi MA. Antipsychotic and antiepileptic drugs in bipolar disorder: the importance of therapeutic drug monitoring. Curren Medicinal Chemistry. 2009; 16: 1463-81.

(9) Zhang G, Jr AV, BArlett MG. Bioanalytical methods for the determination of antipsychotic drugs. Biomed. Chromatogr. 2008 Jul; 22: 671-87.

(10) Mauri M.C. Volonteri L.S. Colasanti A. Fiorentini A. De Gaspari I.F. Bareggi S.R.Clinical Pharmacokinetics of atypical antipsychotics: A critical review of the relationship between plasma concentrations and clinical response. Clin. Pharmacokinet. 2007; 46: 359-388.

(11) Müller MJ, Regenbogen B, Härter S, Eich FX, Hiemke C. Therapeutic drug monitoring for optimizing amisulpride therapy in patients with schizophrenia. J Psychiatr Res. 2007 Oct; 41: 673-9

(12) Müller MJ, Eich Fx, Regenbogen B, Sachse J, Härter S, Hiemke C. Amisulpride doses and plasma levels in different age groups of patients with schizophrenia or schizoaffective disorder. Psychopharmacol. 2009 May; 23: 278-86.

(13) Schwartz JB. The influence of sex on pharmacokinetics.

Clin Pharmacokinet 2003; 42:107-121.

(14) Sparshatt A, Taylor D, Patel MX, Kapur S. Amisulpride-dose, plasma concentration, occupancy and response: implications for therapeutic drug monitoring. Acta Psychiatr Scand. 2009 Dec; 120: 416-28.

(15) Müller MJ, Regenbogen B, Sachse J, Eich Fx, Härter S, Hiemke C. Gender aspects in the clinical treatment of schizophrenic inpatients with amisulpride: a therapeutic drug monitoring study. Pharmacopsychiatry. 2006 Mar; 39: 41-6.

(16) Bergemann N, Kopitz J, Kress KR, Frick A. Plasma amisulpride levels in schizophrenia or schizoaffective disorder. Eur Neuropsychopharmacol. 2004 May; 14: 245:50.

(17) Davis J.M. Chen N. Dose Response and Dose Equivalence of Antipsychotics. J Clin Psychopharmacol. 2004; 24: 192-208.

(18) Kirschbaum KM, et al. Serum levels of aripiprazole and dehydroaripiprazole, clinical response and side effects. World j Biol Psychiatr. 2008; 9: 212-8.

(19) Molden E. Lunde H, Lunder N, Refsum H. Pharmacokinetic variability of aripiprazole and the active metabolite dehydroaripiprazole in psychiatric patients. Ther Drug Monit. 2006 Dec; 28: 744-9.

(20) Hu R, Malhotra A, Pickar D. Predicting Response to Clozapine. CNS Drugs 1998 Apr; 11: 317-326.

(21) Meltzer H. EFfects of six months of clozapine treatment on the quality of life of chronic schizophrenic patients. Hosp Community Psychiatry. 1990; 41:892-897.

(22) Spina E, Avenoso A. Relationship between plasma concentrations of clozapine and norclozapine and therapeutic response in patients with schizophrenia resistant to conventional neuroleptics. Psychopharmacol. 2000 Jan; 148: 83-89.

(23) Jann MW, Grimsley SR, Gray EC, Chang Wen-Ho. Pharmacokinetics and pharmacodynamics of clozapine. Clin. Pharmacokinet 2003 24:161-176.

(24) Edge S. C. Et al. Clozapine drug-drug interactions: a review of the literature. Hum. Psychopharmacol. 1997 12: 5-20.

(25) Tang Y, Mao P. Gender, age, smoking behaviour and plasma clozapine concentrations in 193 chinese inpatients with schizophrenia. Br J Clin Pharmacol. 2007 July; 64(1): 49-56.

(26) Freman DJ, Oyewumi LK. Will routine therapeutic drug monitoring have a place in clozapine therapy? Clin Pharmacokinet. 1997 Feb; 32: 93-100. 
(27) Citrome L. Volavka J. Optimal Dosing of atypical antipsychotics in adults: A review of the current evidence. Harv Rev Psychiatry 2002; 10: 280-291.

(28) Devinsky, O.; Honigfeld, G.; and Patin, J. Clozapine-related seizures. Neurology, 1991; 41 :369-371.

(29) Olesen V. Clozapine serum levels and side effects during steady state treatment of schizophrenic patients: a cross-sectional study. Psychopharmacology. 1995 Feb; $117: 371-8$.

(30) VanderZwaag C. Response of patients with treatment-refractory schizophrenia to clozapine within three serum level ranges. Am J Psychiatry 1996; 153: 1579-158.

(31) Olesen OV. Therapeutic drug monitoring of clozapine treatment. Clin. Pharmacokinet 1998 Jun; 34: 497-502.

(32) Spina E, Avenoso A et al. Relationship between plasma concentrations of clozapine and norclozapina and therapeutic response in patients with schizophrenia resistant to conventional neuroleptics. Psychopharmacol. 2000 Jan; 148: 83-9.

(33) Khan AY, Preskorn SH, et al. Examining concentration-dependent toxicity of clozapine: role of therapeutic drug monitoring. J Psychiatr Pract. 2005 Sep; 11: 289-301.

(34) Szegedi, I. Anghelescu, J.Wiesner. Addition of low dose fluvoxamine to low dose clozapine monotherapy in schizophrenia: Drug Monitoring and Tolerability data from a Prospective Clinical Trial. Pharmacopsychiatry 1999; 32: 148, 153.

(35) Hiemke C, Weigman H, Härtter S, Dahmen N, Wetzel H,. Müller H. Elevated levels of clozapine in serum after addition of fluvoxamine. J Clin Psychopharmacol 1994; 14: 279-281.

(36) Brouwers EE, Söhne M, Kuioers S, Huitema AD, et al. Ciprofloxacin strongly inhibits clozapine metabolism: two case reports. Clin Drug Investig. 2009; 29: 59-63.

(37) Kelly DL, Richardson CM, Yu Y, Conley RR, et al. Plasma concentrations of high-dose olanzapine in a double-blind crossover study. Hum Psychopharmacol. 2006 Aug; 21: 393-8.

(38) Weiss U, Marksteiner J, Kemmler G, Saria A, Aichhorn W, et al. Effects of age and sex on olanzapine plasma concentrations. J Clin Psychopharmacol. 2005 Dec; 25: 570-4.

(39) Aravagiri M, Ames D, Wirshing WC, Marder SR, et al. Plasma level monitoring of olanzapine in patients with schizophrenia: determination by high-performance liquid chromatography with electrochemical detection. Ther Drug Monit. 1997 Jun; 19: 307-13.

(40) Bergemann N, Frick A, Parzer P, Kopitz J, et al. Olanzapine plasma concentration, average daily dose, and interaction with co-medication in schizophrenic patients. Pharmacopsychiatry 2004 Mar; 37: 63-8.

(41) Fellows L, Ahmad F, Castle DJ, Disco LJ, Bulsara MK, Ilett KF. Investigation of target plasma concentration-effect relationships for olanzapine in schizophrenia. Ther Drug Monit $2003 \mathrm{Dec} ; 25$ : 682-9.

(42) Robertson MD, McMullin MM, et al. Olanzapine concentrations in clinical serum and postmortem blood specimens--when does therapeutic become toxic? Forensic Sci 2000 Mar; 45: 418-21.

(43) Lindsay DeVane C. Clinical Pharmacokinetics of Quetiapine: An Atypical Antipsychotic. Clin Pharmacokinet 2001; 40 : 509-522.

(44) Darwish M, Bond M, Hellriegel ET, Youakim JM, Yang R, Robertson P Jr. Investigation of a Possible Interaction Between Quetiapine and Armodafinil in Patients With Schizophrenia: An Open-Label, Multiple-Dose Study. J Clin Pharmacol. 2011 Sep 8. [Epub ahead of print]

(45) Hiemke C, et al. Therapeutic monitoring of new antipsychotic drugs. Ther Drug Monit. 2004 Apr; 26: 156-60. 
(46) Castberg I, Skogvoll E, Spigset O, et al. Quetiapine and drug interactions: evidence from a routine therapeutic drug monitoring service. J Clin Psychiatry 2007 Oct; 28: 1540-5.

(47) Dragicevic A,Trotzauer D, Hiemke C, Muller MJ. Gender and age effects on quetiapine serum concentrations in patients with schizophrenia or schizoaffective disorders, 2005. Lecture presented at the 24th Symposium of the AGNP, 5-8 October, Munich.

(48) Wilson W. A visual Guide to Expected Blood Levels of Long Acting Injectable Risperidone in Clinical Practice. Journal of Psychiatric Practice 2004 Nov; 10: 393-401.

(49) Eresshefsky et al. Comparision of the effects of different routes of antipsychotic administration on pharmacokinetics and pharmacodynamics. J Clin Psychiatry 2003; 64: 18-23.

(50) Olesen OV, Licht RW, Thomsen E, Bruun T, Viftrup JE, Linnet K. Serum concentrations and side effects in psychiatric patients Turing risperidone therapy. Ther Drug Monit Aug; 1998: 380-4.

(51) Lane HY, Chiu WC, Chou JC. Risperidone in acutely exacerbated schizophrenia: doping strategies and plasma levels. Clin Psychiattry, 2000 Mar; 61 : 209-14.

(52) Aveosos A, Facciolá G, Salemi M. Determination of Risperidone and its major metabolite 9-OH-Risperidone in human plasma by reversed-phase liquid chromatography with ultravioleta detection. Journal Chromatogr B Biomed Sci Appl. 2000; 746: 173-181.

(53) Spina E. Avenoso A. and cols. Relationship between plasma risperidone and $9 \mathrm{OH}$ risperidone concentrations and clinical response in patients with schizophrenia. Psychopharmacology. $2001 \mathrm{Jan}$; $153: 238-43$.

(54) Ereshefsky L. Mannaert E. Pharmacokinetic profile and clinical efficacy of long acting risperidone: Potential benefits combining an atypical antipsychotic and a new delivery system. Drugs in $\mathrm{R}$ and D. 2005; $6: 129-137$.

(55) Riedel M, Schwarz MJ, Strassnig M, Spellmann L, Müller-Arends A, Weber K, et al. Risperidone plasma levels, clinical response and side effects. Eur Arch Psychiatry Clin Neurosci. 2005.

(56) Mauri MC, Laini V. Long Term Treatment of chronic schizophrenia with risperidone: a study with plasma levels. Eur. Psychiatry 2001; 16:57-63.

(57) Volonteri LS, Cerveri G, De Gaspari IF, Bali ML, Rolandi ML, Mencacci C, et al. Longacting injectable risperidone and metabolic ratio: a possible index of clinical outcome in treatment-resistant schizophrenic patients. Psychopharmacology (2010) 210: 489-497.

(58) Lane HY, Chiu WC, Chou JC, Wu ST, Su MH, Chang WH. Risperidone in acutely exacerbated schizophrenia: dosing strategies and plasma levels. J Clin Psychiatry. 2000 Mar; 61 : 209-14.

(59) Taylor D. Risperidone long-acting injection in practice, more questions than answers? Acta Psychiatr Scand. 2006 Jul; 114 : 1-2.

(60) Nyberg S, Eriksson B, Oxenstierna G, Halldin C, Farde L. Suggested minimal effective dose of risperidone based on PET-measured D2 and 5-HT2A receptor occupancy in schizophrenic patients. Am J Psychiatry. 1999 Jun; 156 : 869-75.

(61) Kane J, Canas F, Kramer M, Ford L, Gassmann-Mayer C, Eerdekens M, et al. et al Treatment of schizophrenia with paliperidone extended-release tablets: A 6-week placebo-controlled trial. Schizophr Res 2007; 90:147-161.

(62) Kramer M, Simpson G, Macciulis V et al Paliperidone extended-release tablets for prevention of symptom recurrence in patients with schizophrenia. J Clin Psychopharmacol. 2007; $27: 6-14$.

(63) Nazirizadeh Y, Vogel F, Bader W, Haen E, Pfuhlmann, Hiemke C, et al. Serum concentrations of paliperidone versus risperidone and clinical effects. Eur J Clin Pharmacol $2010 ; 66$ : 797-803. 
(64) Farde L, Nordström AL, Wiesel FA, Pauli S, Hallidin C, Sedvall G. Positron emission tomographic analysis of central D1 and D2 dopamine receptor occupancy in patients treated with classical neuroleptics and clozapine. Relation to extrapyramidal side effects. Arch Gen Psychiatry 1992; 49: 538-544.

(65) Kapur S, Zipursky R, Jones C, Remington G, Houle S Relationship between dopamine D(2) occupancy, clinical response, and side effects: a double-blind PET study of first-episode schizophrenia. Am J Psychiatry 2000; 157: 514-520.

(66) Gründer G, Carlsson A, Wong DF. Mechanism of new antipsychotic medications: occupancy is not just antagonism. Arch Gen Psychiatry 2003; 60: 974-977.

(67) Nyberg S, Eriksson B, Oxenstierna G, Halldin C, Farde L Suggested minimal effective dose of risperidone based on PET measured D2 and 5-HT2A receptor occupancy in schizophrenic patients. Am J Psychiatry 1999, $156: 869-875$.

(68) G, E, Mann S, Kapur S et al A PET study evaluating dopamine D2 receptor occupancy for long-acting injectable risperidone. Am J Psychiatry, 2006163 :396-401.

(69) Doran A, Obach S, Yasgar AS, Zhang C. The impact of p-glycoprotein on the disposition of drugs targeted for indications of the central nervous system: evaluation using the MDR1A/1B knockout mouse model. Drug Metab Dispos. 2005; 33:165-174.

(70) Kirschbaum KM, Henken S, Hiemke C, Schmitt U Pharmacodynamic consequences of Pglycoprotein-dependent pharmacokinetics of risperidone and haloperidol in mice. Behav Brain Res. 2008; $188: 298-303$.

(71) Farde L, Wiesel FA, Halldin C, Sedvall G. Central D2-dopamine receptor occupancy in schizophrenic patients treated with antipsychotic drugs. Arch Gen Psychiatry 1988; 45: 71-6.

(72) Vernaleken I, Fellows C, Janouschek H, Bro“cheler A, Veselinovic T, Gründer G, et al. Striatal and extrastriatal D2/3 receptor binding properties of ziprasidone: a PET study with $[18 \mathrm{~F}]$ fallypride and [11C] raclopride. Int Neuropsychopharmacol. 2010 Aug; 13: 951-60.

(73) Miceli JJ, Anziano RJ, Robarge L, Hansen RA, Laurent A. The effect of carbamazepine on the steady-state pharmacokinetics of Ziprasidone in healthy volunteers. Br J Clin Pharmacol 2000; 49: Suppl 1:65S-70S.

(74) Vogel F, Gansmüller R, Leiblein T, Dietmaier O, Wassmuth H, Hiemke C, et al. The use of ziprasidone in clinical practice: Analysis of pharmacokinetic and pharmacodynamic aspects from data of a drug monitoring survey Eur Psychiatry 2009; 24: 143-148. 